\title{
Strain Rate Imaging in Normal and Reduced Diastolic Function: Comparison with Pulsed Doppler Tissue Imaging of the Mitral Annulus
}

\author{
Asbjørn Støylen, MD, Stig Slørdahl, PhD, Gunnar K. Skjelvan, MD, Andreas Heimdal, PhD, \\ and Terje Skjaerpe, PhD, FESC, Trondheim, Norway
}

\begin{abstract}
Objectives: The pixel velocity values obtained by color Doppler tissue imaging (DTI) can be processed to velocity gradients as a measure of longitudinal strain rate with a technique termed strain rate imaging (SRI). Color mapping of strain rate does show the spatial-temporal relations of the diastolic phases. The phases of early filling and late filling during atrial systole can be seen to consist of a stretch wave in the myocardium, propagating from the base to the apex. Diastolic function is characterized by both peak strain rate and propagation velocity of this wave. The goals of this study were to establish normal values for these measurements and to study the changes with minimal diastolic dysfunction.

Methods: Twenty-eight healthy control subjects and 26 patients with hypertension and normal systolic function were studied. The patients had normal blood pressure on treatment, normal ejection fraction, minimal hypertrophy, and moderately prolonged deceleration and isovolumic relaxation times. Realtime SRI color cineloops, ordinary echocardiography
\end{abstract}

\section{BACKGROUND}

The longitudinal motion of the heart shows a descent of the base toward the apex during systole, with a

From the Department of Cardiology, University Hospital of Trondheim (A.S., T.S.); Institute of Physiology and Biomedical Engineering, Norwegian University of Science and Technology (S.S., A.H.); and Trondheim Heart Centre (G.K.S.); Trondheim, Norway.

Dr Heimdal is presently affiliated with Advanced Methods GE Vingmed Ultrasound, Trondheim, Norway.

Supported by a grant from the Norwegian Council for Cardiovascular Disease and the Norwegian Research Council.

Reprint requests: Asbjørn Støylen, MD, Department of Cardiology, University Hospital of Trondheim, N-7006 Trondheim, Norway (E-mail: a-stoe@online.no).

Copyright (C) 2001 by the American Society of Echocardiography. $0894-7317 / 2001 / \$ 35.00+0 \quad 27 / 1 / 110375$

doi:10.1067/mje.2001.110375 and Doppler recordings, and pulsed wave DTI from the mitral ring were acquired and processed.

Results: Patients showed a reduction of systolic and early diastolic tissue velocities and strain rates and no significant increase in late diastolic tissue velocity and strain rate. Propagation velocity of diastolic strain during both early and late filling phases was reduced in the patients. The combination of changes in peak strain rate and propagation velocity of strain rate corresponded with changes in DTI.

Conclusion: Diastolic deformation of the ventricle can be shown as a complex series of events, with temporal sequences in the ventricle. The peak strain rate and the propagation velocities of strain rate can describe the two main diastolic events: early and late filling. In reduced diastolic function, both are reduced during early filling. The velocities of the mitral ring are the result of this combination. This adds information about the physiology and pathophysiology of diastole. (J Am Soc Echocardiogr 2001; 14:264-74.)

reverse movement during the phases of early filling (E) and atrial systole (A), whereas the apex remains virtually stationary throughout the heart cycle. Both longitudinal M-mode and Doppler tissue imaging (DTI) of the mitral annulus have demonstrated this. ${ }^{1-}$ 7 Thus the velocities increase from zero at the apex to a maximum at the base of the ventricle for all phases, ${ }^{8}$ with a longitudinal velocity gradient as shown in Figure 1. It can be shown that the longitudinal velocity gradient is a measure of the rate of longitudinal deformation, or strain rate. 9,10 The unit of strain rate is centimeters per second per centimeter, or $s^{-1}$, and the values of strain rate can be displayed in the same manner as velocities: as color mapping or digital curves, as shown in Figure 2.This method is termed strain rate imaging (SRI). 9,10 In SRI, velocities are converted to regional velocity differences, showing regional systolic dysfunction in 


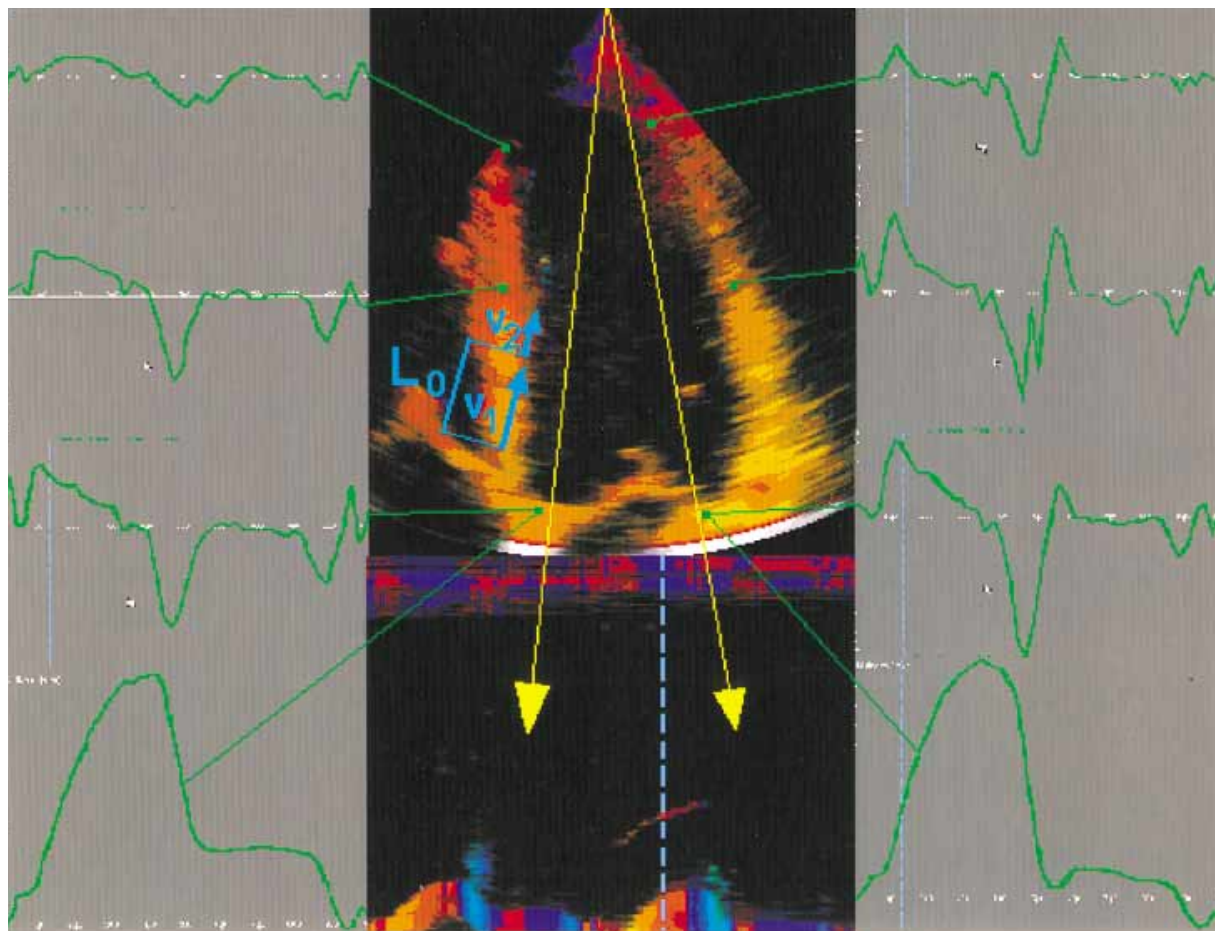

Figure 1 Motion of the heart throughout the heart cycle as shown by tissue Doppler imaging. A systolic frame from color Doppler imaging with the red color of velocities toward the probe is shown. The digital velocity values from the indicated points of the ventricle have been extracted and are displayed as velocity curves shown to the sides, clearly illustrating decreasing velocities from the base to the apex. All curves are simultaneous from the same heart cycle. Below the 2-dimensional picture are reconstructed M-mode images through the mitral ring. The M-mode image is similar to the integral curve from the velocity of the mitral ring, shown below the velocity curves. The picture also shows the longitudinal velocity gradient $(\mathrm{VG})$, that is, velocity difference per length unit: $\mathrm{VG}=\left(\mathrm{V}_{2}-\mathrm{V}_{1}\right) / \mathrm{L}_{0}=\Delta \mathrm{V} / \mathrm{L}_{0}$. Linear or Lagrangian strain $(\varepsilon)$, is relative to length change: $\varepsilon=\Delta \mathrm{L} / \mathrm{L}_{0}$ where $\Delta L$ is length change, and $L_{0}$ is length at baseline. Strain rate is strain per time unit: $\mathrm{SR}=\varepsilon / \Delta \mathrm{t}=\Delta \mathrm{L} / \mathrm{L}_{0} / \Delta \mathrm{t}=\Delta \mathrm{L} / \Delta \mathrm{t} / \mathrm{L}_{0}=\Delta \mathrm{V} /$ $\mathrm{L}_{0}=\mathrm{VG} . \mathrm{L}_{0}$ is exaggerated for clarity.

coronary disease $\mathrm{10}^{10,11}$ and also temporal distribution of deformation in normal ventricles (Figure 3). The velocities caused by contraction of more apical segments or by translation of the whole heart are subtracted. Strain rate imaging provides quantitative measurement of local deformation rates, whereas color mapping provides semiquantitative data and information about the spatial-temporal relations between events in the ventricle during the heart cycle (Figures 4 and 5). The temporal resolution of the postprocessed $\mathrm{M}$-mode data is equal to the frame rate in 2-dimensional recording: up to 130 frames/s when acquiring color DTI images of the whole left ventricle.

The anatomic M-mode image of color SRI reveals a complex pattern (Figures 4 and 5). In our experience, isovolumic contraction seems to occur nearly simultaneously from base to apex, followed by a short, equally simultaneous recoil. Shortening during ejection starts virtually simultaneously as well, except possibly in the apex. Lengthening during isovolumic relaxation generally is seen first in the midwall, and at the same time, reciprocal shortening is seen in neighboring segments, whereas stretching starts later in the apex. Mmode or DTI of the mitral ring can demonstrate a slight lengthening of the whole ventricle during this phase, mainly in the basal parts (Figures 4, 6, and 7). Because SRI only measures longitudinal deformation, the temporal sequence of actual events should be interpreted with caution. In addition, SRI is subject to misinterpretation as a result of angle deviation, ${ }^{9-11}$ and this effect is especially pronounced in the apex. Previous experience with magnetic resonance (MR) has demonstrated that the relaxation starts mainly at the apex, ${ }^{12}$ but as an "untwisting" rather than as a longitudinal deformation.

The main diastolic deformation of the ventricle, however, occurs during the early (E) and late (A) filling phases. Filling of the ventricle progresses as a wave from the mitral annulus to the apex, ${ }^{13,14}$ and so 


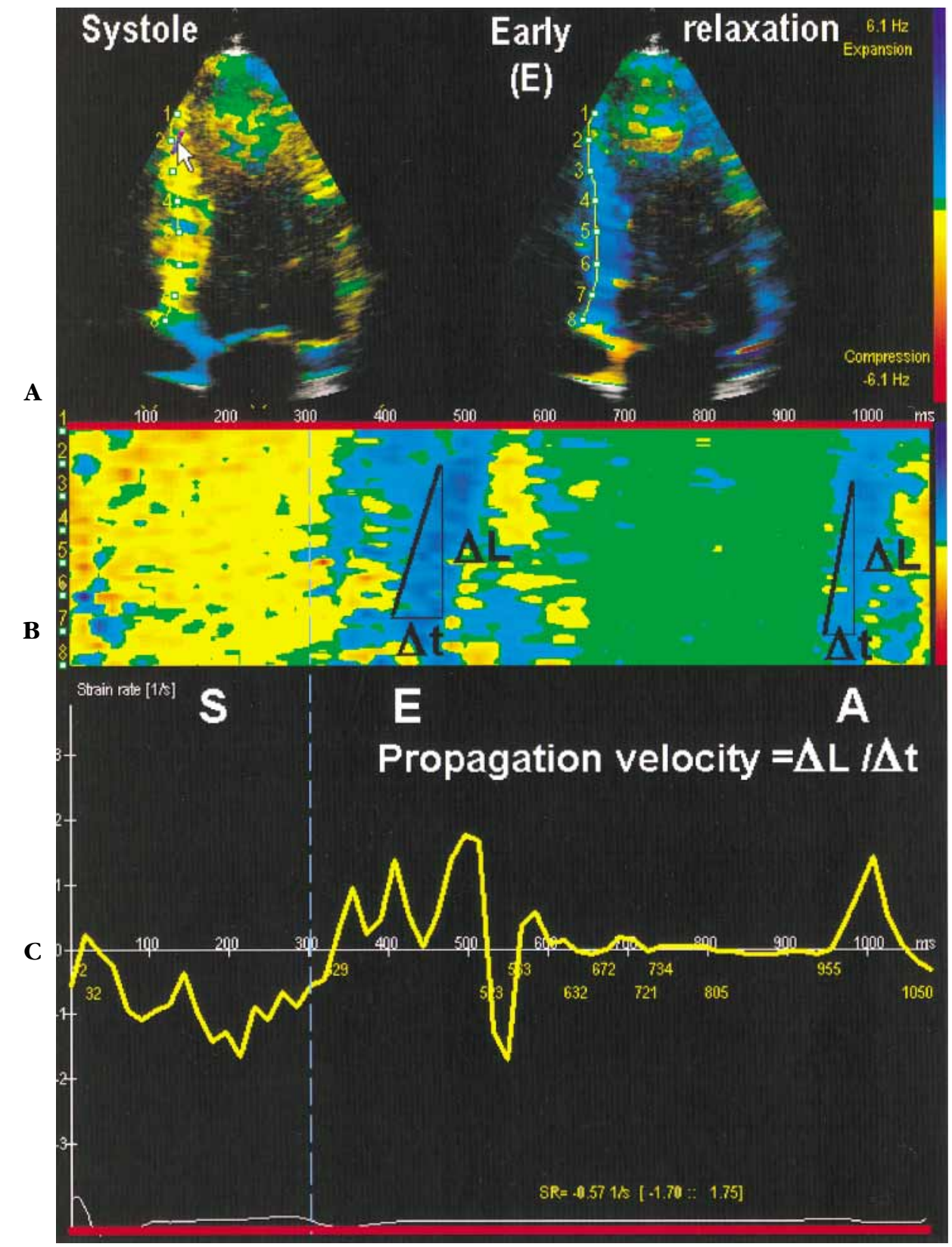

Figure 2 Strain rate imaging from a healthy subject, illustrating the color mapping of strain rate and the principle of the longitudinal M-mode technique. A, Still frames from systole (left) and early filling (right), showing negative strain rate (systolic shortening) as yellow to red colors and positive strain rate (diastolic lengthening) in cyan to blue colors, whereas periods of no strain are shown in green. The color legend is reproduced to the right. A curved M-mode image is obtained in the septum. B, The resulting M-mode image from the same cineloop of one heart cycle. The numbers to the left refer to the numbers on the 2-dimensional images and are included for orientation only, bearing no relation to points of velocity measurements. C, The strain rate curve from the point of the arrow on the systolic frame, and the electrocardiogram for reference. Both color and curve show negative strain rate during systole $(S)$ and positive strain rate during early filling $(E)$ and atrial systole $(A)$. The phases of early and late relaxation can be seen to propagate from the base to the apex as a wave of stretching. The principle of propagation velocity measurement as the slope $\Delta \mathrm{L} / \Delta \mathrm{t}$ is shown here, even though distances must be measured from a straight $\mathrm{M}$-mode line for reasons explained in the text. 


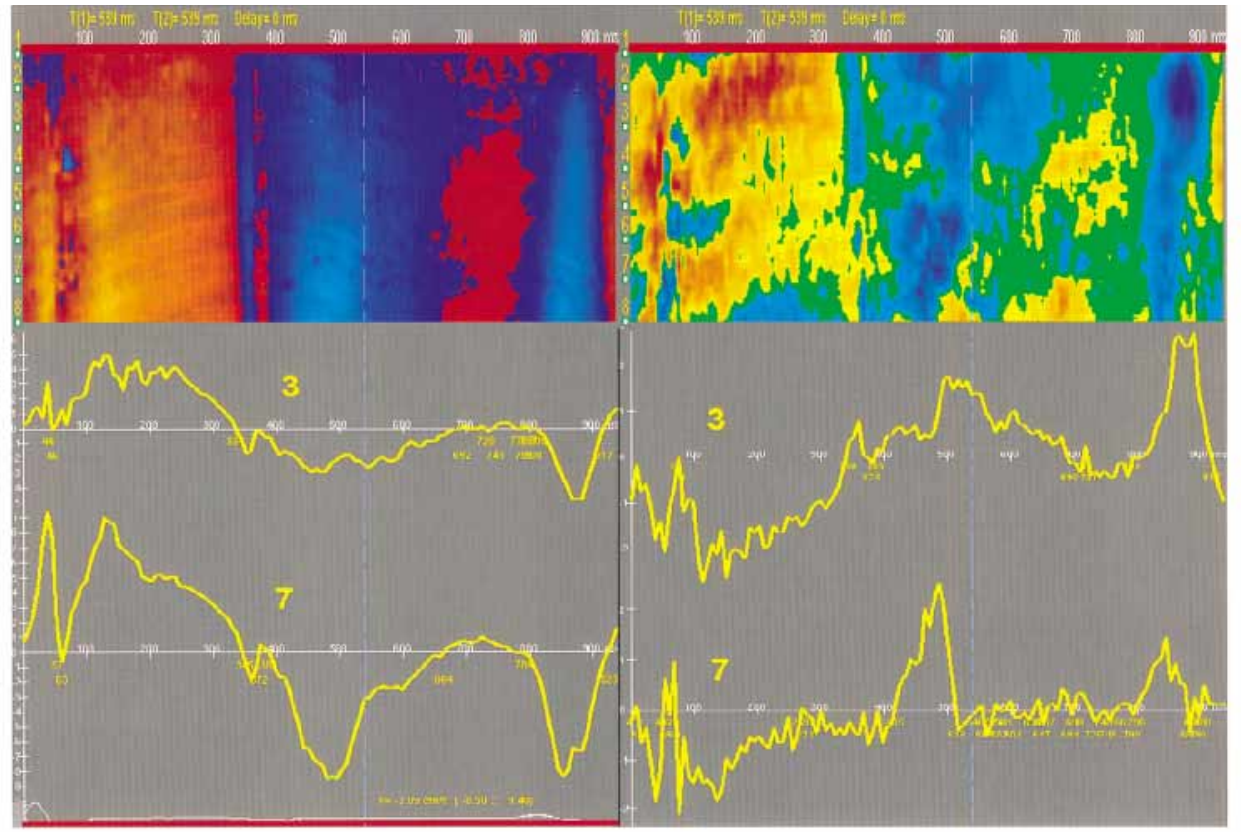

\section{Real-time DTI}

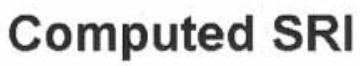

Figure 3 Processing of strain rate data from tissue velocities. The orientation of the M-mode image is as in Figure 2: apex on top, base at the bottom. The cineloop was acquired as a color Doppler tissue image, and the unprocessed Doppler tissue imaging (DTI) data are shown to the left, with the velocity curves from points 3 and 7 . The strain rate image to the right is processed off-line from the same cineloop without changing the position of the M-mode line. Converting the velocities to velocity gradients demonstrates the temporal inequalities of diastolic strain in a normal ventricle, with delay of early and late relaxation at the apex in relation to the base. The apparent decrease in peak early diastolic strain rate from base to apex shown in the picture is caused by random variations, as shown by statistical analysis. SRI, Strain rate imaging.

does the stretching and thinning of the wall (Figure 7). The propagation velocity of this stretching wave can be measured as shown in Figure 2. Propagation velocity of the $\mathrm{E}$ wave is consistently lower than that of the A wave. The $\mathrm{E}$ and $\mathrm{A}$ waves of the mitral annulus seen by pulsed DTI represent the resulting longitudinal deformation rates of the whole ventricle during the filling phases. The stretching waves can be seen to return from the apex to the base in Figure 4. When an anatomic M-mode line is drawn around the whole ventricle as in Figure 5, this may be seen as a continuation of the propagation of the stretch wave around the apex and down the opposite wall. This results in minor oscillations of the mitral ring after the main diastolic events ( $\mathrm{E}$ and $\mathrm{A}$ waves) as shown in Figures 4 and 7 . The impact of this return wave on ventricular geometry appears to be minor as seen by DTI. However, it shows the propagation of the filling phases along the ventricular wall to be more than simply a reflection of flow propagation, and probably reflects the interaction of load and stiffness of the left ventricular wall.

The E/A ratio of peak annulus velocity and annulus motion is decreased with a reduced diastolic function, as illustrated in Figure 7. This change has been shown to be less preload dependent than the E/A ratio of mitral flow. $6,15-17$ The peak velocity of the mitral annulus is determined not only by the rate of deformation (the local strain rate), but also by the propagation of the stretch wave. The slower the propagation, the less simultaneous the stretching of the different parts of the ventricle and the lower the peak velocities of the mitral ring. The present study is an investigation of the relation of the strain rate, strain propagation velocity, and mitral velocities in normal and reduced diastolic function. 


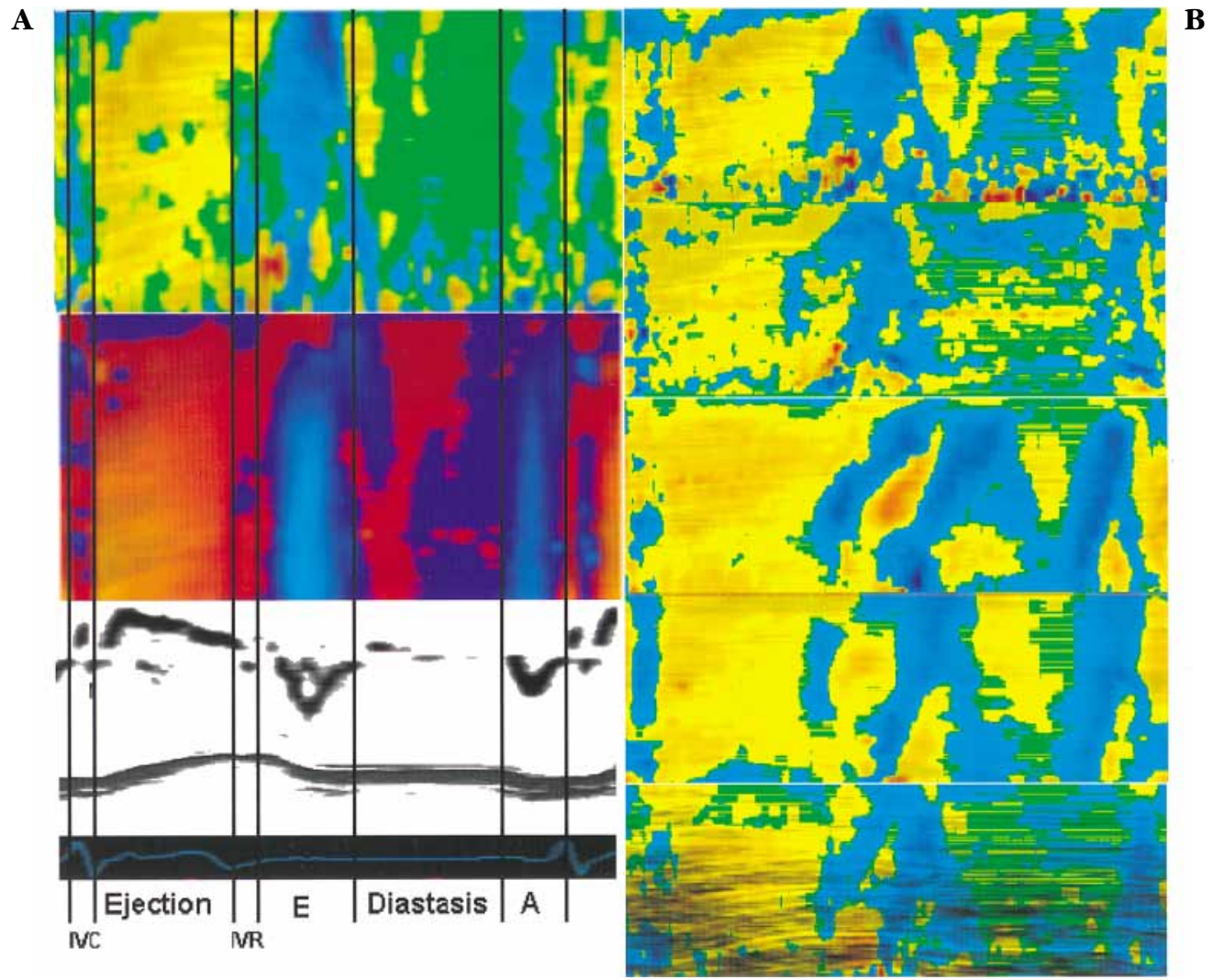

Figure 4 The phases of the heart cycle. A, The relation of the phases with different methods. Top, Longitudinal M-mode image from the septum of a healthy subject (orientation as in Figure 2). Center, The same M-mode image in color Doppler tissue imaging (DTI). Bottom, The DTI and M-mode curves from the septal part of the mitral ring. Isovolumic contraction (IVC) is seen as a brief, nearly simultaneous shortening, followed by a short recoil, an ejection as a phase of prolonged shortening, and isovolumic relaxation (IVR) as a phase of lengthening, with a short recoil. The early filling phase (E), corresponding to the $\mathrm{E}$ wave of the mitral flow, is seen as a wave of stretching that propagates from the base to the apex, and this phase results in the E wave of the DTI curve of the mitral ring. The stretch wave is followed by short recoil, propagating from the apex to the base, resulting in a small oscillation in the DTI curve. The phase of late filling during atrial systole $(A)$ behaves like the early filling phase. $\mathbf{B}$, Longitudinal M-mode images from 5 different healthy subjects, showing the considerable variation of the strain rate imaging pattern, especially in the isovolumic relaxation. The stretch waves can be seen to return from the apex to the base in all 5 samples (see Figure 5 ).

\section{METHODS}

\section{Study Subjects}

Twenty-eight healthy control subjects (7 women, 21 men) without any evidence of heart disease and 26 patients with hypertension (10 women, 16 men) were examined. Informed consent was obtained.The patients participated in the Losartan Intervention for Endpoint Reduction in Hypertension (LIFE) study and the LIFE echocardiographic substudy. ${ }^{18}$ They were selected for the LIFE study according to electrocardiographic criteria for left ventricular hypertrophy, randomized to either losartan or atenolol in a blinded design, and given additional antihypertensive medication of other types as needed. The acquisition of additional SRI and DTI data was done following a controlled schedule in accordance with study protocol after 1 to 2 years of treatment in the study. No patients had regional dysfunction by echocardiography, and no patients had a pseudonormal filling pattern apparent from comparison of mitral flow curves and DTI curves from the mitral ring. ${ }^{15-17}$ The echocardiographer was blinded to all treatments. Subject characteristics of the groups are summarized in Table 1 and show a significant, if slight, hypertrophy, no dilatation, normal systolic function, and reduced diastolic function (delayed relaxation) by deceleration time (dec-t), isovolumic relaxation time (IVR), and E/A ratio reversal. The patients were significantly older than the controls and had significantly higher blood pressure. 


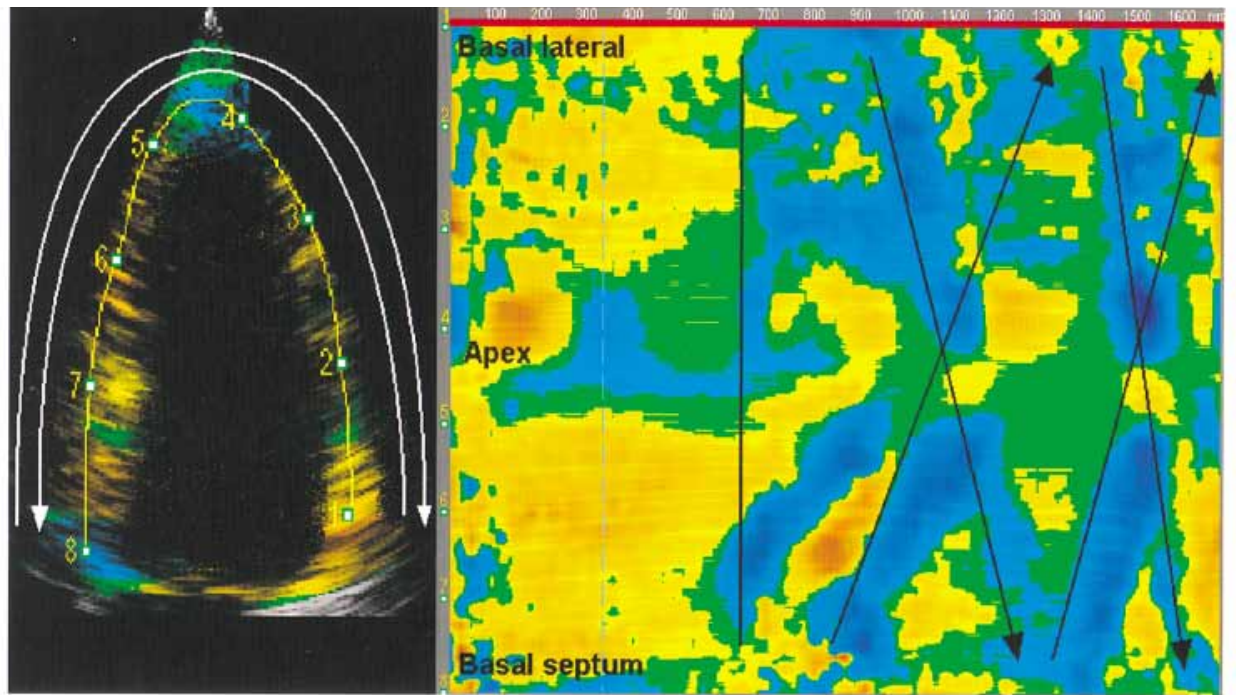

Figure 5 Strain rate pattern in the whole ventricle. A curved M-mode line is placed in the wall from the base of the lateral wall, through the apex, and down to the base of the septum in a 4-chamber view. The numbers show the orientation of the $\mathrm{M}$-mode line on the 2-dimensional frame. The numbers on the $\mathrm{M}$ mode line refer to the same points. Apex is in this view in the middle, whereas the base is on the top and bottom. The isovolumic relaxation is more or less simultaneous in both walls; early and late relaxation are seen to propagate from the base to the apex, but to continue past the apex toward the base of the opposite wall as indicated by the arrows. In the apex, strain values are reversed for geometric reasons. ${ }^{9-11}$

Table 1 Subject characteristics

\begin{tabular}{lcccccccccc}
\hline & $\begin{array}{c}\text { Age } \\
(\mathbf{y})\end{array}$ & $\begin{array}{c}\mathbf{E F} \\
(\mathbf{\%})\end{array}$ & $\begin{array}{c}\text { HR } \\
(\mathbf{b p m})\end{array}$ & $\begin{array}{c}\mathbf{B P} \\
(\mathbf{m m ~ H g})\end{array}$ & $\begin{array}{c}\text { IVSd } \\
(\mathbf{m m})\end{array}$ & $\begin{array}{c}\text { LVDd } \\
(\mathbf{m m})\end{array}$ & $\begin{array}{c}\text { Dec-t } \\
(\mathbf{m s})\end{array}$ & $\begin{array}{c}\text { IVR } \\
(\mathbf{m s})\end{array}$ & $\begin{array}{c}\text { MVE } \\
(\mathbf{c m} / \mathbf{s})\end{array}$ & $\begin{array}{c}\text { MVA } \\
(\mathbf{c m} / \mathbf{s})\end{array}$ \\
\hline Controls & $40(14)$ & $56(6)$ & $63(11)$ & $125 / 77(14 / 14)$ & $8(1)$ & $57(5)$ & $183(32)$ & $77(15)$ & $74(13)$ & $53(14)$ \\
Patients & $65(6)$ & $55(6)$ & $61(14)$ & $153 / 85(18 / 6)$ & $10(2)$ & $53(11)$ & $252(48)$ & $103(19)$ & $70(20)$ & $74(19)$ \\
$P$ value & $<.001$ & NS & NS & $<.01$ & $<.001$ & NS & $<.001$ & $<.001$ & NS & $<.001$ \\
\hline
\end{tabular}

Group averages with standard deviations in parentheses and $P$ values for differences. EF, Ejection fraction (biplane Simpson); $H R$, heart rate; $B P$, blood pressure; IVSd, diastolic thickness of intraventricular septum (M-mode); LVDd, diastolic diameter of left ventricle (M-mode); Dec-t, deceleration time of mitral flow $\mathrm{E}$ wave; $I V R$, isovolumic relaxation time; $M V E$, peak velocity of $\mathrm{E}$ wave in mitral flow; $M V A$, peak velocity of A wave; NS, not significant.

\section{Echocardiography}

A specially programmed System FiVe scanner (GE Vingmed Ultrasound, Horten, Norway) with a 2.5-MHz phasedarray transducer was used. Strain rate imaging cineloops of all 3 standard apical planes were acquired in real time and transferred to a personal computer for analysis with a dedicated software application. The frame rate was 70 to 100 frames/s, and the offset length $\left(\mathrm{L}_{0}\right.$ in Figure 1$)$ was 5 to 10 $\mathrm{mm}$. Peak systolic strain rate (PSRs) and peak strain rate during early (PSRe) and late (PSRa) filling, from the strain rate curves shown in Figure 3, were measured in all 16 standard segments of the left ventricle. ${ }^{19}$ Global averages for the whole ventricle of each person were computed. Longitudinal M-mode lines along the walls were reconstructed from the 2-dimensional cineloops. Because the curvature of the curvilinear M-mode drawing shown in Figure 2 is subjectively chosen, distances are varied. For this reason, propagation velocities were measured along a straight-line M-mode image, but by the same principle as that illustrated in Figure 2. For this reason also, propagation velocities were only measured in the basal half of the walls, where a straight M-mode line could be placed parallel to the wall. Measurements of linear propagation velocities of strain rate during both filling phases (PVSe and PVSa) were performed in all 6 walls of the 3 standard apical planes, and global averages for the whole ventricle were computed for each person.

Standard echocardiographic recordings in the octave mode were acquired during the same examination and transferred to a Macintosh (Apple Computer Inc, Cupertino, Calif) computer for analysis in EchoPAC (GE Vingmed Ultrasound). Ejection fraction (EF) was measured by modified biplane Simpson's method in apical 4-chamber and long-axis planes. Intraventricular septum (IVSd) and left 


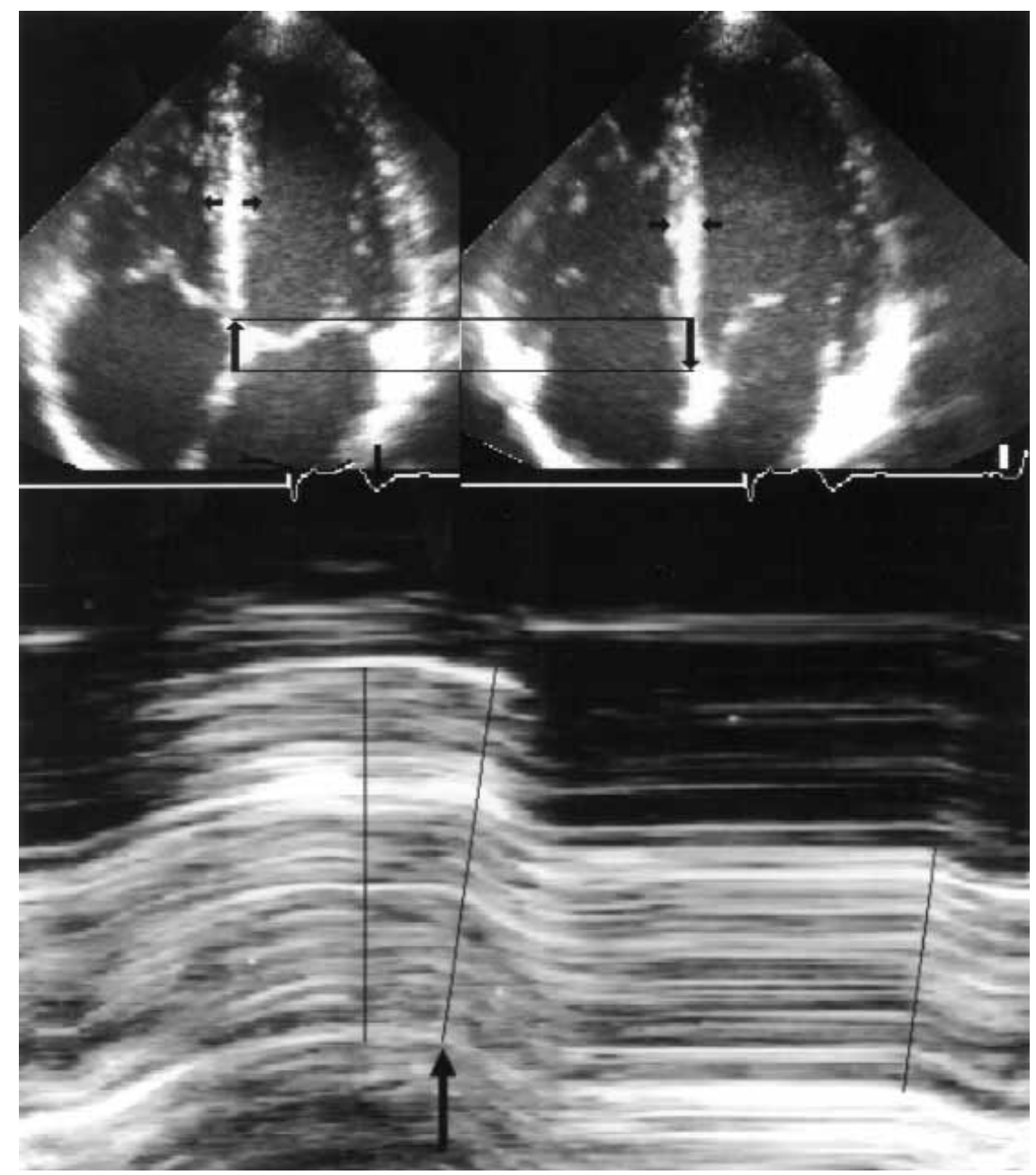

Figure 6 Strain in 2 dimensions. Because heart muscle is incompressible, systolic longitudinal and circumferential shortening has to be balanced by simultaneous wall thickening and diastolic stretching by thinning. As the wall gets thinner by diastolic stretching, the internal diameter increases also. Below, A tissue $\mathrm{M}$-mode image along the septum of a normal ventricle. Isovolumic relaxation at end ejection can be seen by the tissue lines as a slight dip, while the descent of the base during $\mathrm{E}$ and A phases propagates from base to apex.

ventricular internal diameter (LVIDd) were measured in parasternal M-mode. Peak flow velocity of mitral early (PME) and late (PMA) filling and deceleration time of early filling were measured by pulsed Doppler from a position between the tips of the mitral valves. Isovolumic relaxation time was measured with the pulsed Doppler sample volume in a position between the aortic orifice and mitral inflow, with the use of the valve click of aortic closure and the start of mitral flow. Pulsed wave Doppler tissue (pwDTI) curves as shown in Figure 7 were acquired from the mitral annulus in the septal and lateral points of the 4-chamber plane and the anterior and inferior points of the 2-chamber plane. Peak systolic (DTIs) velocity and peak velocity of early relaxation (DTIe) and atrial systole (DTIa) were measured at all 4 points and averaged for each ventricle.

\section{Reproducibility}

Reproducibility of strain rate propagation was only assessed by repeated measurements in the same recordings. Intraobserver variation was assessed by repeated analysis with an interval of 1 year by the principal investigator. Interobserver variation was done with an observer experienced in general echocardiography, but with no previous experience in SRI. The reproducibility of peak strain rate measurements was low because of the high level of noise in the quantitative data, and it was not tested.

\section{Statistics}

$P$ values for differences between patients and controls are 2 -tailed probabilities by Student $t$ test. ( $N$ in the tests represents the number of subjects in each group, not the num- 


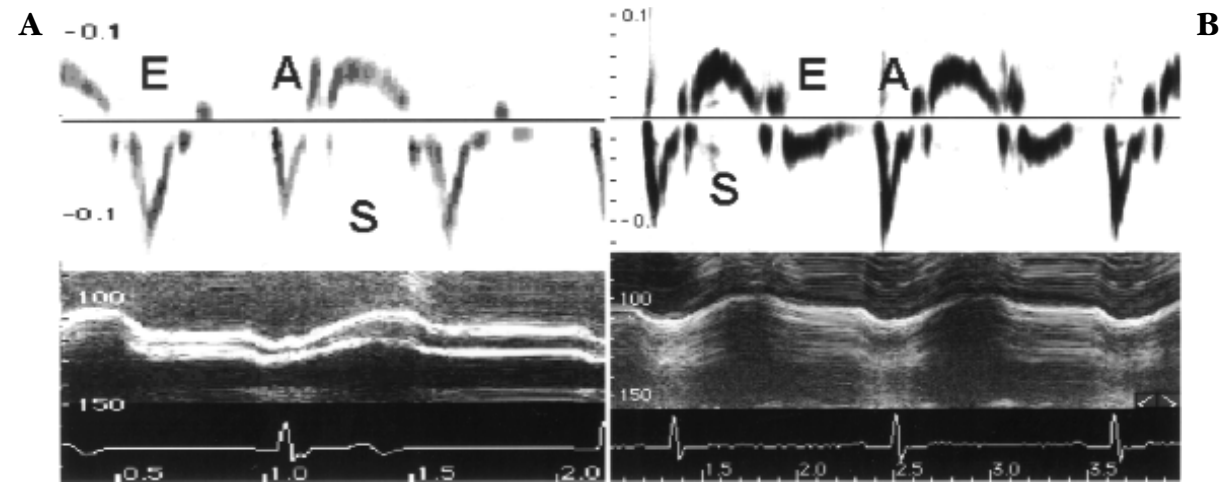

Figure 7 Motion of the septal point of the mitral ring. Top, Tissue velocities by pulsed Doppler. Bottom, Real-time M-mode images through the same point. The phases of systolic shortening $(S)$ and diastolic lengthening during early filling $(E)$ and atrial systole $(A)$ are clearly seen. Isovolumic contraction and relaxation are seen as short velocity changes with recoil in tissue Doppler imaging and as small "notches" in the M-mode curves. A, A healthy subject. B, A patient with delayed relaxation. The reduction in mitral ring motion during the $\mathrm{E}$ phase is evident in both peak velocity and extent of motion.

Table 2 Peak annulus velocities by Doppler tissue imaging, peak strain rate, and strain rate propagation velocity measurements

\begin{tabular}{|c|c|c|c|c|c|c|c|c|}
\hline & $\begin{array}{c}\text { DTIs } \\
(\mathrm{cm} / \mathrm{s})\end{array}$ & $\begin{array}{c}\text { DTIe } \\
(\mathrm{cm} / \mathrm{s})\end{array}$ & $\begin{array}{c}\text { DTIa } \\
(\mathrm{cm} / \mathrm{s})\end{array}$ & $\begin{array}{l}\text { PSRs } \\
\left(\mathbf{s}^{-1}\right)\end{array}$ & $\begin{array}{c}\text { PSRe } \\
\left(\mathbf{s}^{-1}\right)\end{array}$ & $\begin{array}{l}\text { PSRa } \\
\left(\mathbf{s}^{-1}\right)\end{array}$ & $\begin{array}{c}\text { PVSe } \\
(\mathrm{cm} / \mathrm{s})\end{array}$ & $\begin{array}{c}\text { PVSa } \\
(\mathrm{cm} / \mathrm{s})\end{array}$ \\
\hline Controls & $9.5(1.6)$ & $13.1(2.8)$ & $10.2(1.8)$ & $1.40(0.21)$ & $2.22(0.49)$ & $1.49(0.48)$ & $60.0(12.9)$ & $94.0(22.1)$ \\
\hline Patients & $7.0(1.1)$ & $8.2(1.5)$ & $11.0(1.8)$ & $1.15(0.17)$ & $1.46(0.25)$ & $1.66(0.37)$ & $31.6(9.3)$ & $72.0(16.2)$ \\
\hline$P$ value & $<.001$ & $<.001$ & NS & $<.001$ & $<.001$ & NS & $<.001$ & $<.001$ \\
\hline
\end{tabular}

Indicated are standard deviations in parentheses and $P$ values for differences. DTIs, Peak systolic velocity of mitral annulus by pulsed tissue Doppler (DTI); DTIe, peak velocity of mitral annulus E wave; DTIa, peak velocity of annulus A wave; PSRs, peak systolic strain rate in systole; PSRe, peak strain rate in early filling; PSRa, peak strain rate in atrial systole; PVSe, strain rate propagation velocity in early filling; PVSa, strain rate propagation velocity in atrial systole; NS, not significant.

ber of segments or walls.) All significant differences were confirmed by Wilcoxon 2-sample rank sum test, and the 1way analysis of variance was used to test for differences between levels or walls in the ventricle. Reproducibility was calculated according to the Bland-Altman method.

\section{RESULTS}

In control subjects, PSRs could be measured in 382 of 448 possible segments, PSRe in 381 segments, and PSRa in 379 segments. In patients, PSRs could be measured in 337 of 416 possible segments, PSRe in 335 segments, and PSRa in 335 segments. In control subjects, PVSe could be measured in 158 of 168 possible walls and PVSa in 152; in patients, PVSe could be measured in 149 of 156 possible walls and PVSa in 147. Peak DTI and strain rate measurements as well as propagation velocities of strain rate and flow with corresponding $P$ values are summarized in Table 2. The peak tissue velocities in controls correspond to those seen in other studies of healthy populations. ${ }^{20}$ The findings in the patient group are close to those in other studies of delayed relaxation. ${ }^{17}$ There is a significant reduction in both systolic and early diastolic velocities of the mitral annulus in patients. The slight increase in DTIa is not significant. The same results are seen in peak strain rate. Propagation velocity, however, is reduced for both $\mathrm{E}$ and $\mathrm{A}$ waves. There was no difference between basal, midwall, or apical segments in systolic, early, or late diastolic strain rate, nor between different walls in either controls or patients. Propagation velocities showed no difference between walls in controls. The only asymmetrical finding in the patient group was that the PVSa was significantly lower in the anteroseptal wall.

As the two groups were unequal in age, the healthy subjects were segregated according to age in Table 3. Age-related findings in controls were in close agreement with those of other reports of healthy populations. ${ }^{20}$ The differences in systolic and late diastolic peak strain rate and strain rate propagation velocity between controls older than 50 years (average age in this group was 61 years) and patients are still significant, despite the small num- 
Table 3 Measurements of controls according to age

\begin{tabular}{crccccccccc}
\hline $\begin{array}{c}\text { Age } \\
(\mathbf{y})\end{array}$ & \multicolumn{1}{c}{$\begin{array}{c}\text { Mean age } \\
(\mathbf{y})\end{array}$} & $\begin{array}{c}\text { DTIs } \\
(\mathbf{c m} / \mathbf{s})\end{array}$ & $\begin{array}{c}\text { DTIe } \\
(\mathbf{c m} / \mathbf{s})\end{array}$ & $\begin{array}{c}\text { DTIa } \\
(\mathbf{c m} / \mathbf{s})\end{array}$ & $\begin{array}{c}\text { PSRs } \\
(\mathbf{H z})\end{array}$ & $\begin{array}{c}\text { PSRe } \\
(\mathbf{H z})\end{array}$ & $\begin{array}{c}\text { PSRa } \\
(\mathbf{H z})\end{array}$ & $\begin{array}{c}\text { PVSe } \\
(\mathbf{c m} / \mathbf{s})\end{array}$ & $\begin{array}{c}\text { PVSa } \\
(\mathbf{c m} / \mathbf{s})\end{array}$ \\
\hline$<30$ & 9 & 26 & $10.3(0.9)$ & $15.1(1.7)$ & $9.4(1.7)$ & $1.37(0.30)$ & $2.31(0.72)$ & $1.26(0.33)$ & $69.3(13.2)$ & $104.7(19.8)$ \\
$30-50$ & 13 & 41 & $9.5(1.3)$ & $13.1(2.4)$ & $10.2(1.6)$ & $1.42(0.13)$ & $2.20(0.34)$ & $1.45(0.38)$ & $56.0(11.4)$ & $93.0(21.4)$ \\
$>50$ & 6 & 61 & $8.2(2.0)$ & $10.0(2.4)$ & $11.2(2.3)$ & $1.38(0.20)$ & $2.15(0.44)$ & $1.91(0.63)$ & $56.7(9.5)$ & $85.4(33.0)$ \\
\hline
\end{tabular}

Standard deviations are indicated in parentheses. DTIs, Peak systolic velocity of mitral annulus by pulsed tissue Doppler (DTI); DTIe, peak velocity of mitral annulus E wave; DTIa, peak velocity of annulus A wave; PSRs, peak systolic strain rate in systole; PSRe, peak strain rate in early filling; PSR a, peak strain rate in atrial systole; PVSe, strain rate propagation velocity in early filling; $P V S a$, strain rate propagation velocity in atrial systole.

Table 4 Measurements of patients according to heart rate

\begin{tabular}{|c|c|c|c|c|c|c|c|c|c|c|}
\hline $\begin{array}{c}\text { HR } \\
(\mathbf{b p m})\end{array}$ & $\mathbf{N}$ & $\begin{array}{c}\text { Mean HR } \\
\text { (bpm) }\end{array}$ & $\begin{array}{c}\text { DTIs } \\
(\mathrm{cm} / \mathrm{s})\end{array}$ & $\begin{array}{c}\text { DTIe } \\
(\mathrm{cm} / \mathrm{s})\end{array}$ & $\begin{array}{c}\text { DTIa } \\
(\mathrm{cm} / \mathrm{s})\end{array}$ & $\begin{array}{l}\text { PSRs } \\
\left(s^{-1}\right)\end{array}$ & $\begin{array}{l}\text { PSRe } \\
\left(s^{-1}\right)\end{array}$ & $\begin{array}{l}\text { PSRa } \\
\left(s^{-1}\right)\end{array}$ & $\begin{array}{c}\text { PVSe } \\
(\mathrm{cm} / \mathrm{s})\end{array}$ & $\begin{array}{l}\text { PVSa } \\
(\mathrm{cm} / \mathrm{s})\end{array}$ \\
\hline$\leq 60$ & 12 & 49 & $6.7(0.9)$ & $8.8(1.3)$ & $10.4(1.7)$ & $1.11(0.14)$ & $1.43(0.23)$ & $1.47(0.28)$ & $30.2(9.1)$ & $67.2(12.0)$ \\
\hline$>60$ & 14 & 71 & $7.3(1.1)$ & $7.6(1.5)$ & $11.5(1.7)$ & $1.19(0.18)$ & $1.49(0.27)$ & $1.82(0.36)$ & $33.4(10.7)$ & $76.1(18.5)$ \\
\hline$P$ value & & & NS & NS & NS & NS & NS & $<.02$ & NS & NS \\
\hline
\end{tabular}

Indicated are standard deviations in parentheses and $P$ values for differences. DTIs, Peak systolic velocity of mitral annulus by pulsed tissue Doppler (DTI); DTIe, peak velocity of mitral annulus E wave; DTIa, peak velocity of annulus A wave; PSRs, peak systolic strain rate in systole; PSRe, peak strain rate in early filling; PSR a, peak strain rate in atrial systole; PVSe, strain rate propagation velocity in early filling; PVSa, strain rate propagation velocity in atrial systole; NS, not significant.

bers. Because the patients were taking medication, they were separated by resting heart rate (HR) in Table 4 for reasons given in the discussion. There were no differences between the two groups except in late diastolic strain rate. The intraobserver limits of agreement were -29.4 and $32.5 \mathrm{~cm} / \mathrm{s}$; the interobserver limits of agreement were -24.4 and $36.6 \mathrm{~cm} / \mathrm{s}$.

\section{DISCUSSION}

\section{Patients}

Patient and control groups were different in several respects. The age is higher in the patient group. This means that some changes are related to age, as shown by the findings categorized by age in Table 3 , but they are still significantly more pronounced in the patient group than in the older controls, whose age is comparable.

The most important aspect of the patient group is that they had only moderate diastolic dysfunction (class 1, delayed relaxation ${ }^{17}$ ) without confounding factors such as dilation or systolic dysfunction. The hypertrophy was moderate, even if significant, and the tendency for subsequent reduced left ventricular diameter was not significant.

The main advantage of examining patients on treatment is that the opportunity exists to minimize possible effects of an afterload increase. However, the difference in blood pressure is significant, so the afterload is still slightly increased in the patient group.

The main disadvantage of examining patients on treatment is that the treatment itself may have an effect on myocardial function. Because the two treatments (angiotensin II antagonist versus beta-blocker) are radically different, direct effects of medication on myocardial function would presumably show up as differences between treatment groups. Even though individual treatments cannot be identified, sorting the patients by HR increases the proportion of those using a beta-blocker in the low-HR group as it increases the proportion of those using an angiotensin II antagonist in the other. Because no differences exist in systolic or early diastolic function between the two HR groups, it may be a reasonable assumption that the observed differences between patients and controls are not caused by medication. The only difference seen in PSRa, which is lower in the lowHR group, may be a direct effect of HR itself, due to the longer diastasis. It is not even significant if the Bonferroni correction for the number of variables tested is applied.

\section{Measurements}

The systolic peak strain rates seem virtually simultaneous from the base to the apex. The average length of the ventricle at the time of peak systolic strain rate was about $9 \mathrm{~cm}$. The measured systolic strain rate 
should then correspond to a peak annulus velocity of $12.7 \mathrm{~cm} / \mathrm{s}$ as long as the apex is stationary and the peak strain rate is the same along the wall. This is in excess of the DTI measurements in our study and of findings in previous studies. ${ }^{20,21}$ The digital strain rate data are noisy, as shown by the curves in Figures 2 and 3 , and by the wide standard deviations of peak strain rates in Table 2 . When identifying the peak systolic strain rate, the chosen value will often include a high noise component, contributing to the peak value. True peak strain values will therefore usually be lower than the measured strain rate. Peak strain rates during early relaxation and atrial systole are much farther from being simultaneous, and no such simple relation with annular velocities exists, but the peak strain rates should be assumed to be as noisy.

Because the wall is curved, the straight-line Mmode data may underestimate the true distance of the wall and subsequently the propagation velocity. Therefore, only the basal halves of the walls (where they were reasonably straight) were included in this analysis. Even so, patients with hypertrophy tend to show a more sigmoidal shape of the anterior septum in the long-axis view, so the potential to underestimate distance and velocity is greatest here; the finding of reduced strain rate propagation in this wall in patients is probably an artifact resulting from this. The difference between patients and controls was still highly significant $(P<.001)$ when this wall was omitted from the analysis in both groups. The strain rate propagation velocity was measured as constant, but without any evidence that it really is so, apart from the visual impression from our data. Further analysis may give more detailed information about this. As shown, the reproducibility of propagation velocity is low. At present, both strain rate and propagation velocity have rather low reproducibility, and they must be considered only as prototypes for clinical diagnosis, which at present are better suited for physiologic research. Still, applying color SRI to qualitative analysis does improve the ease of interpretation in regional dysfunction. ${ }^{10,11}$ Further research may increase the precision of this method.

\section{Tissue Function}

The information that relaxation propagates as a wave is new, based on the conversion of velocities to velocity gradients. The propagation of strain rate is a wave of stretching during the filling phases. In a previous study, ${ }^{11}$ wall thickening and semiquantitative strain rate seemed to confer the same information, in other words, that wall shortening is equivalent to wall thickening (Figure 7).The converse also applies: as the wall stretches, it thins, increasing the internal diameter of the ventricle.The strain rate propagation can then be seen as a wave of chamber dilation as well as wall stretching.

The reduction of annular velocity during early filling is a result of both reduced strain rate and loss of simultaneity, caused by the slower propagation. The reduced peak strain rate during this phase may be a direct measure of the reduced relaxation rate, whereas the slower propagation may be a result of the more complicated changes in the stiffness of the myocardium during this relaxation and interaction with local load changes.

During atrial systole, on the other hand, the ventricular myocardium is passive, and both peak strain rate and the propagated stretch wave are results of the atrial "pull" on the mitral ring and the active propagation of pressure and flow caused by atrial systole. The ventricular myocardium is passive during this phase, and the strain rate and strain rate propagation must be the result of the properties of the passive myocardium and the load. The strain propagation during this phase was significantly lower in the patient group, whereas peak tissue velocities and strain rates were nearly unchanged.

In the present application, only longitudinal deformation is considered. As MR has shown, there is a significant "twisting" and "untwisting" motion in the myocardium, ${ }^{12}$ which may give different geometric effects on length changes of the ventricle. In addition, SRI measurements show a greater angular distortion than do mere velocity measurements.9-11

\section{Conclusion}

Doppler tissue imaging and tissue Doppler-derived measurements are sensitive measures of diastolic function. Strain rate imaging shows a complex pattern of deformation during diastole because of its high sampling frequency, compared with all other methods, including MR. The main deformations during early and late filling phases are the result of both local relaxation rate and the propagation of the stretching wave from base to apex. This provides new physiologic and pathophysiologic information. The study demonstrates that both peak strain rate and propagation of stretching are reduced in diastolic dysfunction; the findings are consistent with the findings by pulsed DTI of the mitral annulus. The present application, however, seems to overestimate true peak strain rate. In contrast to regional systolic function, the DTI measurements are quicker and may be more practical for clinical work, whereas the level of detail in SRI data enables deeper insight into diastolic physiology. 
Björn Olstad created the dedicated software for SRI analysis, and he has been constantly developing this in response to technical advancements. Our study nurses, Marit O. Röe and Torild Vigeland Nergaard, have been of invaluable assistance.

\section{REFERENCES}

1. Lundback S. Cardiac pumping and function of the ventricular septum. Acta Physiol Scand Suppl 1986;550:1-101.

2. Jones CJ, Raposo L, Gibson DG. Functional importance of the long axis dynamics of the human left ventricle. Br Heart J 1990;63:215-20.

3. Alam M, Hoglund C, Thorstrand C. Longitudinal systolic shortening of the left ventricle: an echocardiographic study in subjects with and without preserved global function. Clin Physiol 1992;12:443-52.

4. Simonson JS, Schiller NB. Descent of the base of the left ventricle: an echocardiographic index of left ventricular function. J Am Soc Echocardiogr 1989;2:25-35.

5. Blomstrand P, Kongstad O, Broquist M, et al. Assessment of left ventricular diastolic function from mitral annulus motion, a comparison with pulsed Doppler measurements in patients with heart failure. Clin Physiol 1996;16:483-93.

6. Bojö L, Wandt B, Ahlin N-G. Reduced left ventricular relaxation velocity after acute myocardial infarction. Clin Physiol 1998;18:195-201.

7. Rodriguez L, Garcia M, Ares M, et al. Assessment of mitral annular dynamics during diastole by Doppler tissue imaging: comparison with mitral Doppler inflow in subjects without heart disease and in patients with left ventricular hypertrophy. Am Heart J 1996;131:982-7.

8. Wilkenshoff UM, Sovany A, Wigstrom L, et al. Regional mean systolic myocardial velocity estimation by real-time color Doppler myocardial imaging: a new technique for quantifying regional systolic function. J Am Soc Echocardiogr 1998;11:683-92.

9. Heimdal A, Stoylen A, Torp H, et al. Real-time strain rate imaging of the left ventricle by ultrasound. J Am Soc Echocardiogr 1998;11:1013-9.
10. Støylen A, Heimdal A, Bjornstad K, et al. Strain rate imaging by ultrasound in the diagnosis of regional dysfunction of the left ventricle. Echocardiography 1999;16:321-9.

11. Støylen A, Heimdal A, Bjørnstad K, et al. Strain rate imaging by ultrasonography in the diagnosis of coronary artery disease. J Am Soc Echocardiogr 2000;13:1053-64.

12. Rademakes FE, Buchalter MB, Rogers WJ, et al. Dissociation between left ventricular untwisting and filling: accentuation by catecholamines. Circulation 1992;85:1572-81.

13. Brun P, Tribouilly C, Duval A-M, et al. Left ventricular flow propagation during early filling is related to wall relaxation: a colour M-mode analysis. J Am Coll Cardiol 1992;20:420-32.

14. Stugaard M, Steen T, Lundervold A, et al. Visual assessment of intra ventricular flow from colour M-mode Doppler images. Int J Cardiac Imaging 1994;10:279-89.

15. Nagueh SF, Middleton KJ, Kopelen HA, et al. Doppler tissue imaging: a noninvasive technique for evaluation of left ventricular relaxation and estimation of filling pressures. J Am Coll Cardiol 1997;30;1527-33.

16. Sohn D-W, Chai I-H, Lee D-J, et al. Assessment of mitral annulus velocity by Doppler tissue imaging in the evaluation of left ventricular diastolic function. J Am Coll Cardiol 1997; 30:474-80.

17. Farias CA, Rodrigues L, Garcia MJ, et al. Assessment of diastolic function by tissue Doppler echocardiography: comparison with standard transmitral and pulmonary venous flow. J Am Soc Echocardiogr 1999;12:609-17.

18. Dahlof B, Devereux R, de Faire U, et al. The Losartan Intervention For Endpoint reduction (LIFE) in Hypertension study: rationale, design, and methods. The LIFE Study Group. Am J Hypertens 1997;10:705-13.

19. Schiller NB, Shah PM, Crawford M, et al. Recommendations for quantitation of the left ventricle by two-dimensional echocardiography. J Am Soc Echocardiogr 1989;2:358-67.

20. Alam M, Wardell J, Andersson E, et al. Characteristics of mitral and tricuspid annular velocities determined by pulsed wave Doppler tissue imaging in healthy subjects. J Am Soc Echocardiogr 1999;12:618-28.

21. Gulati VK, Katz W, Follansbee WP, et al. Mitral annular descent velocity by tissue Doppler echocardiography as an index of global left ventricular function. Am J Cardiol 1996; 77:979-84. 